\title{
Globe
}

Revue internationale d'études québécoises

\section{Analyse des réseaux sociaux et interdisciplinarité dans les études québécoises}

\section{Michel Lacroix}

Volume 7, numéro 1, 2004

Réseaux et identités sociales

URI : https://id.erudit.org/iderudit/1000828ar

DOI : https://doi.org/10.7202/1000828ar

Aller au sommaire du numéro

Éditeur(s)

Globe, Revue internationale d'études québécoises

ISSN

1481-5869 (imprimé)

1923-8231 (numérique)

Découvrir la revue

Citer ce document

Lacroix, M. (2004). Analyse des réseaux sociaux et interdisciplinarité dans les études québécoises. Globe, 7(1), 11-25. https://doi.org/10.7202/1000828ar d'utilisation que vous pouvez consulter en ligne.

https://apropos.erudit.org/fr/usagers/politique-dutilisation/ 


\title{
Introduction
}

\section{Analyse des réseaux sociaux et interdisciplinarité dans les études québécoises}

\author{
Michel Lacroix \\ Université du Québec à Trois-Rivières
}

Que la vie sociale soit réticulairement organisée, que le rapport aux individus, organisations et institutions soit pour une bonne part déterminé par les réseaux, cela n'est pas, en soi, une découverte récente, comme en témoigne l'ancien adage " dis moi qui tu hantes, je te dirai qui tu es ". Néanmoins, on a pu assister à une véritable foison de textes sur et autour des réseaux au cours des dernières années; en conséquence de quoi, le "networking " ou réseautage est devenu un terme à la mode, qui serait dans certains cas plus approprié comme entrée du Dictionnaire $d u$ québécois instantané ${ }^{-1}$ que dans un ouvrage scientifique : il n'est jusqu'aux stratèges militaires américains qui y ont recours, pour se demander si les réseaux représentent la structure idéale des armées futures².

Cette profusion, qui engendre inévitablement une certaine confusion dans les termes et tend parfois à donner une portée idéologique à la notion de réseau ${ }^{3}$, a malheureusement pour effet de placer sous le signe

1. Benoît Melançon, en collaboration avec Pierre Popovic, Dictionnaire du québécois instantané, Montréal, Fides, 2004, 240 p.

2. Les experts du Pentagone ont ainsi remis au Congrès américain un rapport intitulé Network Centric Warfare: Department of Defense, United States of America, Network Centric Warfare. Report to Congress. 27 July 2001, http://www.defenselink.mil/nii/NCW/ (6 avril 2004).

3. L'idéal du réseau, conçu comme système de relations décentré, égalitaire et spontané, se trouve paradoxalement défendu à la fois par les spécialistes de la "nouvelle économie " et les promoteurs de l'* économie sociale ", les partisans

Michel Lacroix, - Analyse des réseaux sociaux et interdisciplinarité dans les études québécoises ", Globe. Revue internationale d'études québécoises, vol. 7 , $n^{\circ} 1,2004$. 
dẹ la mode un chantier intellectuel extrêmement dynamique, auquel participent des chercheurs de multiples disciplines des sciences humaines, celui de l'étude des réseaux. Or, ce chantier est ouvert depuis plusieurs décennies. Qui plus est, un des principaux axes d'étude, celui de l'analyse des réseaux sociaux, a produit un ensemble de définitions extrêmement précises ${ }^{4}$. Enfin, les acquis accomplis dans le cadre de ce chantier, et tout particulièrement les percées récentes, en font un des champs de recherche importants des études québécoises actuelles. Il importe donc de ne pas confondre les discours qui font du réseau la forme idéale des relations sociales et les travaux qui en font un objet d'étude ${ }^{5}$. On peut cependant juger, comme le fait Vincent Lemieux dans ces pages ${ }^{6}$, que l'importance prise par les recherches sur les réseaux tient à ce que celles-ci répondent à des interrogations actuelles et proposent un regard sur les faits sociaux en phase avec certaines transformations majeures des conceptions occidentales, dont celles touchant à la recomposition des identités sociales. Ainsi, les questionnements sur les

comme les adversaires de la mondialisation économique. Les premiers écrivent par exemple: "The information revolution is favouring and strengthening network forms of organisation. (Global Profile, "Network warfare", http://www.globalprofile.co.uk/53, 6 avril 2004), alors que les seconds veulent aller "vers un réseautage de l'économie sociale et solidaire" (Patrick Develtere, Pascal Laviolette et Emmanuelle Robertz, *Une économie sociale et solidaire à dimension internationale se dessine-t-elle?", http://www.econosoc.org/ publications/internationale.htm, 6 avril 2004).

4. Comme nous l'indiquerons plus bas, l'" analyse des réseaux sociaux " (network studies) est un courant de recherches spécifique au sein des sciences sociales, pourvu d'un appareil notionnel et de techniques d'analyse propres, qui distinguent les travaux qui en résultent des autres filières à l'œuvre dans le chantier sur les réseaux. Ce ne sont donc pas toutes les études sur les réseaux qui peuvent être rangées sous le signe de l'analyse des réseaux sociaux, bien au contraire. Les relations entre ces divers courants de recherche constituent, comme on le verra, un des enjeux des travaux actuels.

5. Cela est particulièrement net en ce qui concerne le traitement réservé au pouvoir, au contrôle, aux conflits et aux hiérarchies. Alors que les premiers ont tendance à opposer catégoriquement les réseaux aux organisations, en faisant des réseaux des lieux libres de contrôle et de conflits, les seconds ont montré qu'il y a aussi des enjeux de contrôle, des conflits entre acteurs, des luttes pour le pouvoir au sein des réseaux.

6. D'autres pistes d'explication sont avancées par celui-ci dans Les réseaux d'acteurs sociaux, Paris, Presses universitaires de France, coll. *Sociologies *, 1999 , p. 3-4. 


\section{ANALYSE DES RÉSEAUX SOCIAUX ET INTERDISCIPLINARITÉ}

vastes catégories transversales comme les classes sociales ou les nations cèdent le pas à celles sur les associations, communautés et autres trames relationnelles entre individus. Dans les mots de Peter V. Marsden, "les réseaux sont omniprésents dans la société contemporaine ${ }^{7}$.

Bien que très diverses, aussi bien pour ce qui est de leurs objets d'étude ou de leurs méthodologies que de leur inscription dans des disciplines et traditions distinctes, les recherches sur les réseaux partagent le postulat qu'Alain Degenne et Michel Forsé placent aux fondements de l'analyse des réseaux sociaux. Défini comme "déterminisme faible ", ce postulat se résume chez eux aux propositions suivantes : "a) la structure ne se réduit pas à une somme d'actions individuelles; b) elle exerce une contrainte mais seulement formelle, qui laisse l'individu libre de ses actes bien que, compte tenu de cette contrainte, tout ne lui soit pas possible ${ }^{8}$. À cet égard, le présent dossier sur les réseaux sociaux cadre parfaitement avec les études du précédent numéro de Globe. Sans être directement en relation avec les objets, théories ou méthodes des auteurs du dossier sur la "Circulation des discours", les spécialistes des réseaux réunis ici participent eux aussi de ce mouvement visant à réévaluer le rôle du sujet dans les changements sociaux.

Le regard sur les structures sociales constitue un second élément de partage conférant unité aux travaux sur les réseaux. Ceux-ci procèdent à un examen du social à partir des relations concrètes entre acteurs, en vertu de l'idée selon laquelle "les phénomènes sociaux peuvent être étudiés comme des réseaux de relations, la société dans son ensemble pouvant elle-même être conçue comme un réseau9". L'importance ainsi

7. Peter V. Marsden, "Social Networks „, Edgar F. Borgatta et Rhonda J. V. Montgomery [éd.], Encyclopedia of Sociology, New York, MacMillan, 2000, p. 2727 [notre traduction]. On peut toutefois penser, à la lumière des travaux historiques sur les réseaux du $x x^{e}$ et du $x x^{e}$ siècles qu'on trouvera ici, que l'importance des réseaux comme facteur explicatif des phénomènes sociaux vaut tout autant pour d'autres périodes.

8. Alain Degenne et Michel Forsé, Les réseaux sociaux. Une analyse structurale en sociologie, Paris, Armand Colin, 1994, p. 11.

9. A. M. Chiesi, "Network Analysis ", Neil J. Smelser et Paul B. Bates [éd.], International Encyclopedia of The Social and Bebavorial Sciences, Oxford, Elsevier, 2001, p. 10502, notre traduction. 
accordée aux actions individuelles déplace le pôle d'observation du côté de la micro-sociologie, bien que l'on tente toujours de partir des observations sur les individus et leurs relations pour dégager des lois expliquant le fonctionnement des structures sociales. Cette volonté d'analyser les rapports entre les parcours individuels et les phénomènes sociaux caractérise les recherches sur les réseaux: "Ainsi, l'étude des réseaux contribue à faire le pont entre les perspectives micro- et macrosociologiques ${ }^{10}$... Comparant-cette approche aux-traditions de l'individualisme méthodologique et du holisme (dont Durkheim serait le fondateur), Degenne et Forsé y vojent l'amorce d'un nouveau paradigme, nommé " interactionnisme structural ${ }^{11}$ ". Moins catégorique, Vincent Lemieux écarte la notion de paradigme, préférant parler du passage d'un schème d'intelligence du social à un autre (du schème causal au schème structural) ${ }^{12}$.

À partir de ces positions communes, plusieurs perspectives sont possibles selon la part laissée aux contraintes sociales transversales, aux identités collectives ou aux mutations historiques de la longue durée. Pour certains, qui voient dans les réseaux une dimension parmi d'autres des phénomènes sociaux, cette approche s'avère conciliable avec une théorie sociologique comme celle de Bourdieu sur les champs, malgré les différences indubitables ${ }^{13}$. Pour d'autres, au contraire, tout ce qui est social peut se ramener, en dernière instance, à des considérations réticulaires ${ }^{14}$. Par ailleurs, du fait que les chercheurs s'intéressant aux réseaux proviennent d'horizons multiples, avec tout ce que cela implique quant aux traditions disciplinaires, aux bagages théoriques ou aux rapports avec les autres disciplines, des écarts sensibles séparent parfois ceux-ci. Enfin, l'extrême variété dans la nature des réseaux observés limite

10. Peter V. Marsden, op. cit., p. 2727 , notre traduction.

11. Alain Degenne et Michel Forsé, op. cit., p. 16.

12. Vincent Lemieux, op. cit., p. 4-6.

13. Cette approche est défendue avec force par Wouter De Nooy, "Fields and Networks: Correspondence Analysis and Social Network Analysis in the Framework of Field Theory ", Poetics, vol. 31, n 5-6, octobre-décembre 2003, p. 305-327.

14. C'est le cas, par exemple, de Degenne et Forsé, Les réseaux sociaux, op. cit. 


\section{ANALYSE DES RÉSEAUX SOCIAUX ET INTERDISCIPLINARITÉ}

quelque peu l'établissement d'un corpus commun et la collaboration active entre chercheurs.

On peut toutefois observer l'esquisse d'une convergence entre les diverses approches, tout particulièrement dans le cas des travaux accomplis dans le cadre des études québécoises. S'ouvre ainsi la possibilité du passage d'un chantier multidisciplinaire mais cloisonné, où il n'y a d'interactions qu'entre quelques disciplines, à une interdisciplinarité plus générale. Ce numéro veut d'ailleurs nourrir le dialogue entre les chercheurs d'horizons multiples; c'est pourquoi on y trouvera convoqués des perspectives disciplinaires aussi diverses que l'histoire intellectuelle, la littérature, la musicologie et la science politique. Les études rassemblées ici montrent que l'on commence à jeter des ponts sur le fossé qui séparait jusqu'à tout récemment les études centrées sur les réseaux culturels ou intellectuels et les travaux issus des sciences sociales. Un des signes les plus clairs de ce rapprochement se trouve ici dans le partage d'un certain nombre de références.

L'inspiration centrale, dans ce numéro comme dans les travaux sur les réseaux en général, est celle de l'analyse des réseaux sociaux (network studies). Fondée il y a plus de trente ans, sous la double influence de la sociométrie et de l'anthropologie culturelle, cette approche s'est imposée depuis comme "l'un des secteurs les plus dynamiques de la sociologie contemporaine ${ }^{15}$ ". Animée d'une double visée, celle de constituer un arsenal de notions permettant de penser et de décrire les réseaux, d'une part, et celle de développer des outils et procédures pour formaliser et traiter mathématiquement les observations des chercheurs d'autre part, l'analyse de réseau a suscité quantité de découvertes majeures, de l'importance des liens faibles dans le parcours des individus au rôle des trous structuraux dans les échanges de ressources en passant par les diverses formes de centralité, les principes de groupabilité et de connexité ou la circulation de l'information ${ }^{16}$. Du fait que l'analyse des

15. Peter Hedström et Richard Swedberg, * Introduction to this Special Issue on Social Newtork Analysis", Acta Sociologica, vol. 37, 1994, p. 327, notre traduction.

16. Pour une présentation synthétique de l'analyse des réseaux, voir Vincent Lemieux, Les réseaux d'acteurs sociaux, op. cit. et Alain Degenne et Michel Forsé, 


\section{REVUE INTERNATIONALE D'ÉTUDES QUÉBÉCOISES}

réseaux sociaux se penche sur les structures de relations sans a priori au sujet des attributs des acteurs ou du "contenu "des liens entre eux, elle a servi à l'étude d'une grande variété de réseaux: familiaux, de voisinage, inter- et intra-organisationnels, de soutien, de diffusion d'idées ou de maladies, de politiques publiques, etc. Une notion, en particulier, celle de capital social, semble promise à un avenir théorique prometteur, car elle offre une interface théorique entre les réflexions de Bourdieu sur les formes de capital (culturel; économique, social et symbolique ${ }^{17}$ ) et les théorisations effectuées dans le cadre de l'analyse des réseaux ${ }^{18}$. De plus, du fait de son fondement métaphorique ${ }^{19}$, elle se prête davantage que les méthodes sociométriques à une utilisation dans les domaines historiques et culturels.

Cette malléabilité, jointe à la solidité de l'armature théorique, a facilité la percée de cette nouvelle approche dans de multiples domaines des sciences sociales ${ }^{20}$, cependant que le formalisme mathématique (plus particulièrement visible dans le recours aux graphes et aux matrices) a pu freiner son intégration aux démarches de ceux qui, en art,

Les réseaux sociaux, op. cit. Pour une présentation détaillée des notions et procédures, voir Ronald S. Burt et Michael J. Minor, Applied Network Analysis a Methodological Introduction, Beverly Hills, Sage, 1983, 352 p., ou Stanley Wasserman et Katherine Faust [éd.], Social Network Analysis: Methods and Applications, New York, Cambridge University Press, coll. "Structural analysis in the social sciences ", 1994, 825 p. Pour un récit de ses origines, voir : John Scott, Social Network Analysis. A Handbook, London, Sage, 2000, 208 p. 17. Pierre Bourdieu, "Les trois états du capital culturel ", Actes de la recherche en sciences sociales, vol. 30, novembre 1979, p. 3-6; * Le capital social. Notes provisoires ", Actes de la recherche en sciences sociales, vol. 31, janvier 1980, p. 23; "Le champ littéraire "Actes de la recherche en sciences sociales, vol. 89, septembre 1991, p. 3-46.

18. Nan Lin, Social Capital. A Theory of Social Structure and Action, Cambridge, Cambridge University Press, coll. "Structural Analysis in the Social Sciences ", 2001, 278 p. ; Nan Lin, Karen Cook et Ronald S. Burt [éd.], Social Capital. Theory and Research, New York, Aldine de Gruyter, 2001, 333 p.

19. Fondement qu'il convient de ne pas oublier, comme le rappelle Cari $\mathbf{L}$. Bankston III, "Social Capital as Process: The Meanings and Problems of a Theoretical Metaphor ", Sociological Inquiry, vol. 72, $\mathrm{n}^{\circ} 2$, printemps 2002, p. 285-317.

20. "It is now seen as a distinct speciality witbin several social science disciplines ", Peter V. Marsden, op. cit., p. 2728. 
en histoire, en littérature ou en musique, cherchent à étudier les réseaux. Plutôt que de recourir aux techniques mises au point pour quantifier les relations et comparer différents réseaux entre eux, un certain nombre de chercheurs, dont ceux intéressés par les réseaux culturels, ont préféré s'inspirer des notions et des principes généraux de l'analyse des réseaux. Les travaux en cours marqueront sans doute plus clairement les avantages et les acquis de cette entreprise. Le recours à la quantification, aux statistiques et à la théorie des graphes est-il vraiment la condition nécessaire d'un étude rigoureuse des réseaux? N'a-t-on pas tendance, dans certains cas, à surévaluer les profits de la formalisation et de l'appareillage technique? N'y a-t-il pas place, aussi, pour des travaux développant une analyse qualitative et historique des réseaux? Comme l'écrit Gérard Fabre dans ce numéro: "les apprentissages interdisciplinaires peuvent être salutaires en tant que garde-fous réciproques : si d'un côté, la conscience de l'historicité prémunit contre les abstractions mathématiques, de l'autre côté, l'effort de construction théorique est rarement vain, il empêche de faire comme si le monde social était transparent et immédiatement accessible à la compréhension ${ }^{21}$. Toutes ces questions doivent encore être débattues pour que s'instaure une interdisciplinarité féconde, réflexive et bidirectionnelle entre l'analyse des réseaux sociaux et les autres pistes de recherches sur les réseaux. Nous pensons pour notre part que les découvertes sur l'historicité des formes et des lieux de sociabilité, les interactions entre capital social et capital symbolique ou l'importance des représentations des sociabilités, principalement dues aux travaux en histoire et en littérature, peuvent nourrir la réflexion des praticiens de l'analyse des réseaux sociaux.

Du fait que la naissance de cette nouvelle voie a eu lieu dans le domaine de la sociologie américaine et que sa percée dans la sociologie française fut relativement tardive, on aurait pu croire que les études québécoises connaîtraient le même retard. Or, il n'en fut rien. Dès les années soixante-dix, une série d'articles sur les réseaux sociaux était publiée, sous la plume de celui qui fut au Québec à la fois un des "maitres fondateurs n de la science politique et le principal pionnier de

21. Voir infra, p. 47. 
l'analyse des réseaux : Vincent Lemieux ${ }^{22}$. Depuis, ses travaux ont inspiré quantité de chercheurs, qui ont exploré à leur lumière de multiples aspects de la société québécoise, dont le soutien social, la recherche d'emploi, les identités locales et l'immigration. Examinant, dans l'article inaugural de ce numéro, la place prise au fil des ans par les études sur les réseaux sociaux dans le domaine de la science politique au Québec, Vincent Lemieux raconte l'histoire de ces rapports, histoire dont il a été un des principaux acteurs. Il indique ainsi les apports de l'analyse des réseaux sociaux à l'étude des rapports entre structures de parenté et clientélisme politique ou des positions des acteurs dans les réseaux d'influence et de mobilisation. Il revient aussi sur l'opposition fondamentale entre appareil (ou organisation) et réseau, qu'il a été l'un des premiers à théoriser. À l'encontre de tous ceux qui nomment réseau toute structure sociale mettant en contact direct des individus ou qui en font un terme trop unilatéralement mélioratif, Lemieux y va d'une mise en garde claire : "la valeur discriminante de cette notion a une valeur à peu près nulle si un réseau en vient à désigner tout ensemble de relations sociales, quel qu'il soit ${ }^{23}$. Il importe donc, de son point de vue, de rendre raison des relations concrètes entre acteurs en fonction d'un spectre de possibilités allant du réseau à l'appareil en passant par le quasi-réseau et le quasi-appareil ${ }^{24}$. Cette lecture des systèmes de relations s'avère d'une grande utilité pour ceux qui souhaitent examiner la façon dont les réseaux évoluent en marge des appareils, au sein des appareils ou s'institutionnalisent pour se transformer eux-mêmes en appareil. Comment ne pas voir tout le parti que l'on peut en tirer dès lors que l'on veut examiner les relations au sein d'un parti politique ou l'évolution d'un groupe littéraire, de la revue de collège à la maison d'édition, pour prendre deux exemples nettement distincts? Lemieux aborde enfin, dans son article, la question des "réseaux de politique publique ", qui susci-

22. Pour une exploration de sa biographie intellectuelle, voir l'entretien réalisé par André-J. Bélanger dans Jean Crête [éd.], Hommage à Vincent Lemieux. La science politique au Québec. Le dernier des mâ̂tres fondateurs, Québec, Presses de l'Université Laval, 2003, p. 11-62.

23. Voir infra, p. 32.

24. En plus de l'article publié dans ce numéro, voir au sujet de cette opposition Vincent Lemieux, Les réseaux d'acteurs sociaux, op. cit., et Vincent Lemieux, Réseaux et appareils, Paris, Maloine, 1982. 
tent de plus en plus l'intérêt des politologues, insistant en passant sur le fait que, quelles que soit la politique publique envisagée, il n'y a jamais une seule coalition, un seul réseau, mais une pluralité de réseaux.

Les enjeux liés aux questions de gouvernance sont aussi abordés par Peter Graefe, qui dans l'article "La topographie des think tanks patronaux québécois. La construction d'un paysage néolibéral " tente de dresser le premier portrait des laboratoires d'idées (think tanks) néolibéraux québécois. Relativement proche de ce pan important de l'analyse des réseaux qu'est le corpus de travaux sur les partages d'acteurs par les conseils d'administration des grandes entreprises (interlocking directorates) ${ }^{25}$, il cherche à mettre au jour les liens entre les laboratoires d'idées, les élites patronales et l'État québécois, tant sur le plan des acteurs ou des ressources financières que sur celui des discours. Il peut ainsi démontrer que l'argent et les acteurs circulent tout aussi facilement que les idéologies, mieux encore, que la circulation d'argent et d'acteurs facilite la propagation de l'idéologie ; tout ceci l'amène à remettre en doute, en conclusion, la résistance proclamée de l'État québécois aux offensives néolibérales. Par ailleurs, Graefe met aussi en évidence l'étroite collaboration de ces laboratoires québécois avec leurs homologues établis ailleurs au Canada, ce qui confirme la thèse à l'effet que les associations patronales sont celles qui réussissent le mieux à coordonner leur action par-delà la frontière Québec-Canada.

Le rôle des réseaux dans la circulation des discours a fait l'objet de quantité d'études chez les historiens de la littérature au cours des dernières années, à la faveur du renouveau considérable qu'ont connus les travaux sur l'épistolaire ${ }^{26}$. Longtemps considérées comme des écrits d'importance secondaire essentiellement utiles à servir de matériau

25. Voir à ce sujet Ronald S. Burt, Corporate Profits and Cooptation : Networks of Market Constraints and Directorate Ties in the American Economy, New York, Academic Press, 1983; Beth Mintz et Michael Schwartz, "Interlocking Directorates and Interest Group Formation ", American Sociological Review, vol. 46, 1981, p. 851-869; William Roy, * The Interlocking Directorate Structure of the United States *, American Sociological Review, vol. 42, 1983, p. 248-257.

26. On peut trouver dans les bulletins de l'Association internationale de recherche sur l'épistolaire un dépouillement détaillé de ces travaux. Parmi ceux ayant été réalisés au Québec, signalons Michel Biron et Benoît Melançon [éd.], 
biographique, les correspondances sont désormais examinées comme registre spécifique d'écriture et comme lieu de rencontre entre activité scripturaire et travail de sociabilité. Ce faisant, de nouvelles perspectives ont été ouvertes, allant du rôle de la lettre dans la mise en place et la perpétuation des relations sociales jusqu'à la représentation de soi et du social en passant par la poétique épistolaire. Les chercheurs en études québécoises ont été nombreux à s'illustrer dans ce domaine de recherche, qui s'est élargi pour inclure aussi les autres genres intimes et la réflexion sur les archives. Parmi ces recherches, on peut signaler celles de Manon Brunet, qui ont contribué, avec celles de Bernard Andrès et de Micheline Cambron ${ }^{27}$, à fonder sur de nouvelles bases la lecture de la littérature québécoise du XIX siècle. Dans la foulée de ses travaux sur l'histoire intellectuelle, sur les problèmes méthodologiques liés à l'étude des réseaux ${ }^{28}$ ainsi que de cette vaste entreprise qu'est la publication intégrale de la monumentale correspondance de Henri-Raymond

Lettres des années trente, Ottawa, Le Nordir, 1996 ; Jacques Blais [éd.], Louis Fréchette épistolier, Québec, Nuit blanche, coll. "Séminaires", 1992 ; Manon Brunet [éd.], Henri-Raymond Casgrain épistolier. Réseau et littérature au XIXe siècle, Québec, Nuit blanche, coll. * Séminaires *, 1995 ; Manon Brunet et Bernard Andrès [éd.], Érudition et passion dans les écritures intimes, Québec, Nota bene, 1999 ; Benoît Melançon, Diderot épistolier: éléments pour une poétique de la lettre au XVII siècle, Montréal, Université de Montréal, 1991 ; Benoît Melançon et Pierre Popovic [éd.], Les femmes de lettres : écriture féminine ou spécificité générique ?, Montréal, Centre universitaire de lecture sociopoétique de l'épistolaire et des correspondances, Université de. Montréal, 1994 ; Benoit Melançon [éd.], Penser par lettre: actes du colloque d'Azay-le-Ferron, mai 1997, Saint-Laurent (Québec), Fides, 1998. Dans les travaux en cours, notons ceux de l'، Initiative interuniversitaire de recherche sur les manuscrits et les archives littéraires *, dirigée par Micheline Cambron et Bernard Andrès.

27. Mentionnons ainsi les recherches du premier sur a L'archéologie du littéraire au Québec, 1760-1840 . (FQRSC et CRSH, 1991-2003) et de la seconde sur - La non-lecture du $x_{x} x^{e}$ siècle québécois ou la constitution d'une vulgate * (CRSH 2001-2003), de même que, parmi les multiples publications, Bernard Andrès et Marc-André Bernier [éd.], Portrait des arts, des lettres et de l'éloquence au Québec (1760-1840), Québec, Presses de l'Université Laval, coll. a La République des Lettres *, 2002 et Micheline Cambron [éd.], Le Journal Le Canadien. Littérature, espace public et utopie (1836-1845), Montréal, Fides, coll. •Nouvelles études québécoises ", 1999.

28. Manon Brunet, * Prolégomènes à une méthodologie d'analyse des réseaux littéraires. Le cas de la correspondance de Henri-Raymond Casgrain ", Voix et images, vol. 27, $\mathbf{n}^{\circ}$ 2, hiver 1992, p. 216-237; Manon Bunet et Pierre Lanthier [éd.], l'inscription sociale de l'intellectuel, Paris/Sainte-Foy, L'Harmattan/PUL, 2000. 
Casgrain, Manon Brunet aborde ici deux réseaux du xIx siècle québécois qui structurèrent la diffusion d'un discours amalgamant religion et littérature. Tous deux influencés par la pensée d'un théologien français important mais méconnu, mgr Jean-Joseph Gaume, ces réseaux se sont dissociés puis opposés l'un à l'autre, au nom de conceptions différentes de la littérature. Leur reconstitution et leur analyse permettent ainsi de plonger au coeur des débats du $\mathrm{Xx}^{\mathrm{e}}$ siècle québécois, au moment où la littérature est en voie d'autonomisation par rapport à la religion, mais aussi de voir que, si les réseaux font circuler les idéologies, leur composition dépend elle-même partiellement des prises de position de chacun des acteurs. Ceci constitue une excellente démonstration de ce que les réseaux intellectuels sont tout à la fois des réseaux d'affinité, basés sur un sentiment d'appartenance, des réseaux de communication, où la propagation de l'information ou de discours est centrale, et des réseaux de mobilisation, où les acteurs, pour atteindre leurs buts, agissent sur les autres acteurs du réseau, par le biais de la coopération ou en imposant leur contrôle ${ }^{29}$.

Avec l'article de Julie Roy, "Des réseaux en convergence. Les espaces de la sociabilité littéraire au féminin dans la première moitié du $\mathrm{XIX}^{\mathrm{e}}$ siècle ", c'est toujours le $\mathrm{XIX}^{e}$ siècle québécois qui est étudié, mais dans une perspective qui s'attache davantage aux formes et aux lieux de sociabilité. Dans la lignée des recherches qui tentent de réévaluer la place des femmes dans l'histoire littéraire, elle examine des pratiques qui, en marge des principaux lieux de formation du champ littéraire, ont permis aux femmes de s'engager dans des activités d'écriture. Les correspondances et salons de Marguerite Lacorne et de Louise-Amélie Panet, de même que les albums de Marie-Reine-Josephte Belleau et d'Adèle Berthelot-Lafontaine, à la frontière de l'intime (sphère socialement dévolue, alors, à la femme) et du public, permettent une diffusion " contrôlée " conduisant à une certaine reconnaissance sociale comme "productrices culturelles". Certes limitées aux heureuses représentantes de l'élite sociale québécoise, l'étude de ces pratiques lève le voile sur le rôle de ces femmes dans la sociabilité de l'époque et dans les échanges

29. Sur ces différents types de réseaux, voir Vincent Lemieux, Les réseaux d'acteurs sociaux, op. cit. 
de textes, de discours, de dessins ou de partitions musicales qui caractérisent la première moitié du $\mathrm{xx}^{\mathrm{e}}$ siècle. L'analyse des albums, où l'on recueillait poèmes, témoignages et portraits signés par des membres du réseau personnel, nous introduit ainsi à un type d'œuvres fort peu connues mais révélatrices. Lire la lettre écrite pour l'album d'Adèle Berthelot-Lafontaine par le chevalier de Lorimier le matin du jour de son exécution nous plonge au coeur d'une entreprise remarquable de mémorialiste politique. Julie Roy accomplit dans son article une convaincante démonstration des pouvoirs heuristiques de l'étude des réseaux : mieux que maintes autres perspectives, celle-ci permet d'explorer la "multiplexité "ou multidimensionnalité de la trame des relations sociales. Peutêtre est-elle plus forte en ce début de $\mathrm{xIX}^{\mathrm{e}}$ siècle où les champs ne sont pas nettement séparés les uns des autres, mais elle n'est pas simplement caractéristique d'une époque, bien au contraire. En effet, les liens entre acteurs sociaux outrepassent bien souvent les frontières institutionnelles ou sociales entre classes, cultures, sexes ou secteurs d'activité. Toutefois, d'un réseau à l'autre, d'une culture à l'autre et d'une époque à l'autre, cette propension à la multidimensionnalité varie grandement, aussi doiton encourager les études qui contribueront à éclairer cet aspect de la vie sociale québécoise.

C'est entre autres choses ce qu'accomplit aussi Gérard Fabre, dans son article intitulé "Un arc transatlantique et sa tangente ou comment se dessine un réseau intellectuel franco-québécois? ". Ce sont les frontières nationales qui s'y trouvent au premier plan, mais on y voit aussi s'entrecroiser réseaux diplomatiques, économiques, familiaux, mondains, politiques et universitaires. Posant d'emblée en introduction à son étude l'importance des réseaux comme lieu de transmission de savoir et d'ancrage du sentiment d'appartenance, il aborde le cas du réseau francoquébécois cristallisé autour de ces "silhouettes nodales" que furent Édouard Montpetit et André Siegfried. Reconstituant, à partir du parcours biographique de ces deux chefs de file, la naissance des sous-réseaux qui, de chaque côté de l'Atlantique, étaient tournés l'un vers l'autre, Fabre met en relief le rôle joué par les contacts universitaires entre l'École libre des sciences politiques de Paris et l'École des hautes études commerciales de Montréal, lesquels assurent un recrutement au réseau, en plus d'analyser les contacts diplomatiques qui alimentent les visites 


\section{ANALYSE DES RÉSEAUX SOCIAUX ET INTERDISCIPLINARITÉ}

réciproques et les liens concrets entre acteurs. Mais, plus profondément encore, c'est le partage de valeurs communes qui permet aux liens de se solidifier et de se multiplier; le réseau franco-québécois en question adhère ainsi à une même vision de l'intellectuel-expert de questions sociales, à une même croyance dans les mérites d'un libéralisme modéré et d'un christianisme tempéré. Malgré ces facteurs, le réseau ne survivra pas aux bouleversements entrainés par la Crise économique, puis par la Seconde Guerre mondiale. Ce n'est d'ailleurs pas un des moindres mérites de cet article que d'examiner tout à la fois la naissance et la désagrégation de ce réseau, soulevant ainsi au passage d'importantes questions quant aux diverses phases dans l'évolution des réseaux.

L'article de Gérard Fabre témoigne éloquemment du dynamisme actuel de l'histoire intellectuelle au Québec comme en France ${ }^{30}$ et des découvertes qui lui sont redevables quant aux relations entre le Québec et la France ${ }^{31}$, alors que de leur côté Manon Brunet et Julie Roy illustrent le développement considérable connu par les études sur les réseaux et les sociabilités littéraires. Peu à peu, grâce à ces travaux, l'histoire des réseaux littéraires et intellectuels québécois devient plus systématique et cumulative. Les différents secteurs de la vie culturelle n'en sont toutefois pas au même degré de développement ; ainsi, peu de spécialistes se sont penchés sur les réseaux ayant marqué l'histoire du cinéma, des arts graphiques, du théâtre ou de la musique. Poursuivant un programme de recherches visant précisément à combler les lacunes au sujet des réseaux musicaux $^{32}$, Marie-Thérèse Lefebvre dresse tout d'abord, dans * Le milieu

30. Voir à ce sujet les collections des revues Mil neuf cent et Mens, ainsi que François Dosse, La marche des idées: bistoire des intellectuels, bistoire intellectuelle, Paris, Éditions La Découverte, 2003; Damien-Claude Bélanger, Sophie Coupal et Michel Ducharme [éd.], Les idées en mouvement. Perspectives en bistoire intellectuelle et culturelle du Çanada, Québec, Presses de l'Université Laval, 2004.

31. Signalons, entre autres textes, Catherine Pomeyrols, Les intellectuels québécois, formations et engagements, 1919-1939, Paris, L'Harmattan, 1996 et Yvan Lamonde, Allégeances et dépendances, Québec, Nota bene, 2001.

32. Du projet - Histoire de la vie musicale au Québec entre 1903 et 1953 : étude des réseaux et de leur impact sur la création et la diffusion de la musique canadienne ", subventionné par le CRSH, est issu, entre autres : "Les SoiréesMathieu (1930-1935) ", Les Cahiers des Dix, n 57, 2003, p. 85-118. 
musical québécois et ses réseaux. Le cas des bourses du gouvernement attribuées aux compositeurs (1919-1929)", un portrait des principaux réseaux articulant le milieu musical québécois au début du $\mathrm{xx}^{\mathbf{e}}$ siècle, avant d'analyser de façon détaillée l'influence de ceux qui furent à l'œuvre derrière le choix des bourses accordées aux compositeurs par le gouvernement du Québec. On voit ainsi s'opposer deux coalitions qui tentent de diriger à leur façon les décisions d'Athanase David, alors secrétaire de la province (poste comprenant des attributions équivalentes à celles d'un ministre de la culture), coalitions qui s'opposent quant aux choix des candidats ou quant à l'opportunité de faire financer par l'État le Conservatoire de musique de Montréal. Ainsi, les modernistes, défenseurs de la candidature de Rodolphe Mathieu, ont davantage d'entrées auprès de David et du gouvernement de Louis-Alexandre Taschereau, alors que leurs adversaires plus traditionnalistes, zélateurs d'une musique " canadienne * et défenseurs d'Eugène Pelletier, s'avèrent plus nombreux. Chacun de ces camps parvient à soutirer l'appui de David, mais ce sera finalement Claude Champagne, fort de ses appuis dans les deux réseaux, qui aura le parcours institutionnel le plus prestigieux. Dans l'affrontement entre ces camps, qui recoupent assez étroitement les clivages littéraires contemporains entre exotiques et régionalistes, on voit apparaître quelques-uns des principaux acteurs de la vie culturelle de l'époque, dont Olivar Asselin, Claude-Henri Grignon, Arthur Laurendeau, Édouard Montpetit et Olivier Maurault. Ainsi, dans cette étude comme dans celles de Manon Brunet, de Julie Roy et de Gérard Fabre, on met en lumière l'aspect composite, transmédiatique des réseaux : chez Marie-Thérèse Lefebvre, quelques-uns des rameaux des réseaux universitaires, littéraires ou politiques se trouvent impliqués dans les conflits qui déchirent le milieu musical, alors que chez Gérard Fabre ce sont les réseaux diplomatiques, mondains et familiaux qui jouent sur la structure des réseaux universitaires et que chez Julie Roy et Manon Brunet on assiste à un enchevêtrement profond des réseaux littéraires, mondains et politiques ou littéraires et religieux. Tout ceci témoigne abondamment de l'intérêt qu'il y aurait à ce que des projets collectifs et interdisciplinaires se penchent sur les réseaux sociaux de l'histoire québécoise ${ }^{31}$. Les pistes

31. Soulignons à ce sujet la démarche entreprise en ce sens par le projet intitulé "Penser l'histoire de la vie culturelle québécoise ", titulaire d'une subvention du 


\section{ANALYSE DES RÉSEAUX SOCIAUX ET INTERDISCIPLINARITÉ}

ouvertes par ces études sont nombreuses. Comment les réseaux des différents milieux s'interpénètrent-ils? De quelle manière ces réseaux recomposent-ils les clivages entre classes, cultures, langues, régions ou générations ? Comment et dans quelles circonstances disparaissent-ils ou s'institutionnalisent-ils dans des appareils formels? Quel est leur rôle dans la circulation des discours et la construction des identités collectives? Quand on aura pu analyser tout cela, sur d'assez longues périodes, à propos de domaines différents et de réseaux multiples, un grand pas aura été fait dans la connaissance de la vie culturelle et sociale québécoise.

FQRSC, auquel œuvrent Micheline Cambron, Michèle Dagenais, Gilbert David, Gagnon, François Hébert, Ollivier Hubert, Serge Lacasse, Marie-Thérèse Lefebvre, Lucie Robert, Denis Saint-Jacques et l'auteur de ces lignes. 